\title{
Research of Optical Cable Overhead Laying Automation
}

\author{
Hong Liang* Mi Zhang Jianmin Peng Dajian Jian Le Chang Wei Zhou \\ College of Science Subjects of Communication University of China \\ lianghong@cuc.edu.cn
}

Keywords: Optical Cable Overhead Laying Vehicle; Automation

Abstract:fiber-optic communication has become the primary means of information transmission nowadays. As the carrier of information transmission, the fiber optic cable has a variety of laying methods such as the direct burial, pipelines, overhead laying and so on. In this paper, starting from the current status of the overhead cable laying, a new automated laying theory is discussed and the concrete models are given.

\section{Introduction}

In recent years, with the rapid development of data communication networks, the fiber optic cables are used more and more for reasons of bandwidth, security, etc. The fiber optic cable construction specifications, construction methods, laying methods and special equipment are booming. ${ }^{[1,2]}$ At present, the common fiber optic cable laying methods are: direct burial, pipelines and overhead laying. The various laying methods are under development from the direction of manual laying to the mechanization and automation. ${ }^{[3,4]}$

\section{Research Status of Optic Fiber Cable Overhead Laying Machine}

In the early 1980s, one category of fiber optic cable designed for use in the power sector had been developed and applied. They are not self-supporting fiber optic cables, but require the appropriate equipment and methods to make them attached to the ground or phase line of the power circuits. ${ }^{[5,6]}$ When laying the fiber optic cables, the cable tray is moved along the tower road on the ground or the cables are expanded on the ground in advance. The fiber optic cables are guided from the ground to the air, which will be laid along with the winding machine. The annealed stainless steel wire with $\varphi 1.0-1.2 \mathrm{~mm}$ should be used to bind the cables on the overhead ground wire. This method is mature, simple and effective. Fig. 1 is the schematic of domestic optic fiber cable overhead laying winding machine.

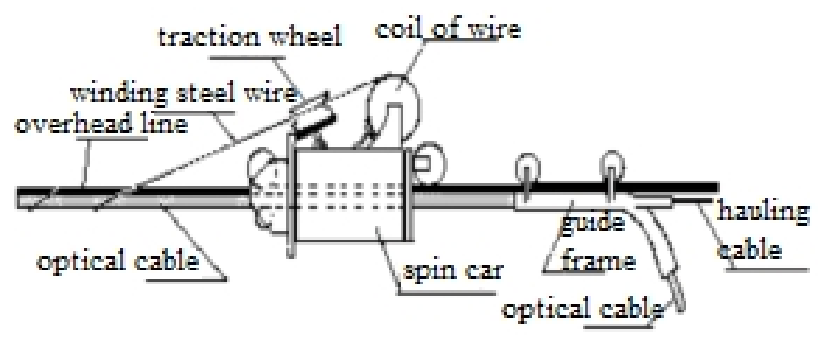

Fig.1 Schematic of domestic optic fiber cable overhead laying winding machine

The structure of the optic fiber cable overhead laying winding machine developed and applied abroad is relatively more complex. ${ }^{[7,8]}$ When laying the fiber optic cables, the cable tray is hanging in the air together with the winding machine, which is driven by the tractor on the lines, and it is 
less affected by topography; it is changed to use coated aramid wire instead of steel wire and it is bifilar winding. The allowed tensile deformation of the aramid wire is much larger than the steel wire, and in case of line disconnection, no power line short circuit will occur; such equipment is relatively more complex to use, which needs to be imported or hired currently, and the cost is very expensive.

No matter it is the domestic, imported or rented winding machine, when conducting the cable laying construction, the cable winding tension control is the key. If the tension is too small, with the swinging and sag changes of the overhead lines, the cable will converge at the lowest point of the sag, so that the winding pitch at the lowest point can become while the one at both sides of the tower can become large, resulting in the uneven loading of the optical fiber, and thus forming the transmission attenuation changes and shortening of the life of the optical fiber (when the ratio of the optic fiber core diameter and the fiber core bend diameter is less than 50, the amount of light transmission has begun to decline, and so is the coupling transmission efficiency). If the winding tension is too large, the fiber-optic will receive excessive tension in the construction, which will not only cause additional fiber attenuation, but fiber breakage in severe cases. In addition, the overhead cable laying winding machine is designed based on borrowing the power lines, which is very different with the current routing environments especially designed for laying the cable. In particular, the imported winding machine is required to hang the cable on the hanging line together. As the cable tray weight is too heavy, the handing line for overhead laying fiber optic cable can hardly stand, it is not widely applied in the country. However, in the international arena, such technology has not been eliminated and given up, which is still applied within the larger scope.

At present, the common domestic outdoor overhead cable laying is still done manually, which is time-consuming, difficult in construction and low in efficiency, and cannot meet the needs of rapid development of optical fiber communications. There is an urgent need for the invention of a kind of new overhead cable laying automation equipment.

\section{Design of Overhead Optic Fiber Cable Laying Vehicle}

The theoretical design of overhead optic fiber cable laying vehicle has been completed, and was awarded the national invention patent in 2013, (Patent No.201010502267.2) The optic fiber cable overhead laying vehicle consists of the power traction mechanism, optic fiber cable transmission mechanism, optic fiber cable hook lifting mechanism, optic fiber cable hook clamping mechanism, intelligent control agencies and auxiliary bodies. Fig. 2 shows the functional structure of the optic fiber overhead laying vehicle.Fig. 3 shows structural schematic of optic fiber cable overhead laying vehicle. The basic functions of the various parts will be introduced as follows.

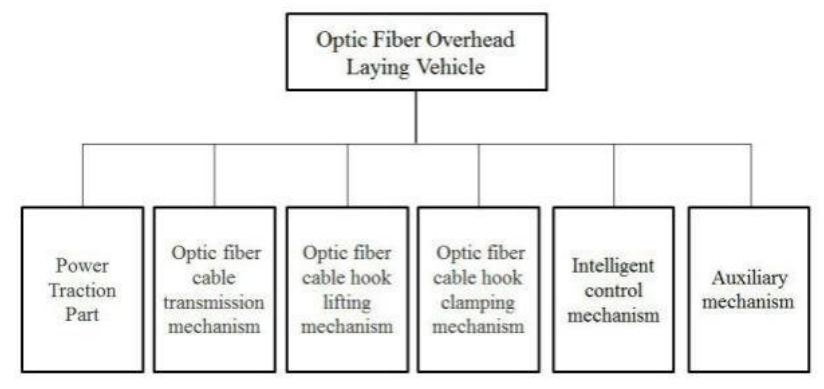

Fig. 2 the Functional Structure of Optic Fiber Overhead Laying Vehicle 


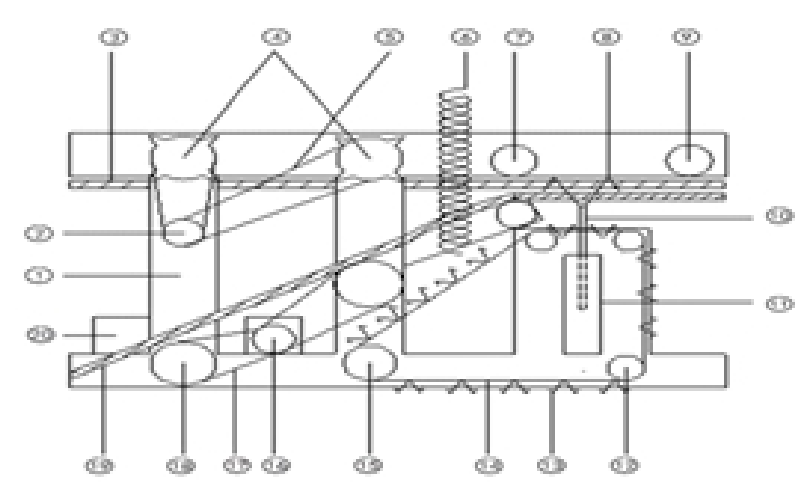

(1) car body frame cable (2) traction motor (3) steel strand (4) star pulley (5) drive belt (6) locating spring (7) locating pulley(8)cable hook(9)compress pulley(10)fiber optic cable hook sucker(11) cable hook lift (12) conveyor guide wheel (13) cable hook line (14) conveyor (15) hook transmission motor (16) cable transmission motor (17) cable transmission belt (18) cable transfer guide wheel (19) optical cable (2) control host

Fig.3 Structural Schematic of Optic Fiber Cable Overhead Laying Vehicle

\section{Power Traction Part}

The traction sheave bears the whole weight of the optic fiber cable overhead laying vehicle and it is hung on the hanging line of the optic fiber cable laid. The traction sheave is rotated as it is driven by the motor, so as to provide the moving power of the optic fiber overhead cable laying vehicle (the traction force should not exceed $80 \%$ of the maximum allowable tension of the cable). The optic fiber cable hanging wire support frame at the top of the pole is a place that must be crossed by the laying vehicle, so the traction sheave is designed to the pulley wheels with the radial shaped grip hook, which can make the cable laying vehicle smoothly pass the support frame, and keep the vehicle from derailing when it is crossing the optic fiber cable hanging wire supporting frame.

2.Optic fiber cable transmission mechanism

The optic fiber cable transmission pulley is a pulley block, which leads the optic fiber cable into the cable overhead laying vehicle and makes the cables laid beneath the hanging line, preparing for the addition of cable hooks. Optic fiber cable transmission cable pulley is driven by the motor, and the optic fiber is transmitted to the predetermined position.

3.Optic fiber cable hook lifting mechanism

The cable hook is preset on the cable hook conveyor belt, and the cable hook suction cup will hook up one cable hook to move up, and hold the cable to fit the preset position beneath the cable hanging wire, and the fiber optic cable hook sucker will drive the cable hook to rotate $90^{\circ}$ clockwise; the cable hook sucker will reset downwards, and then hang on the cable hook hanging wire.As shown in the Fig. 4 .

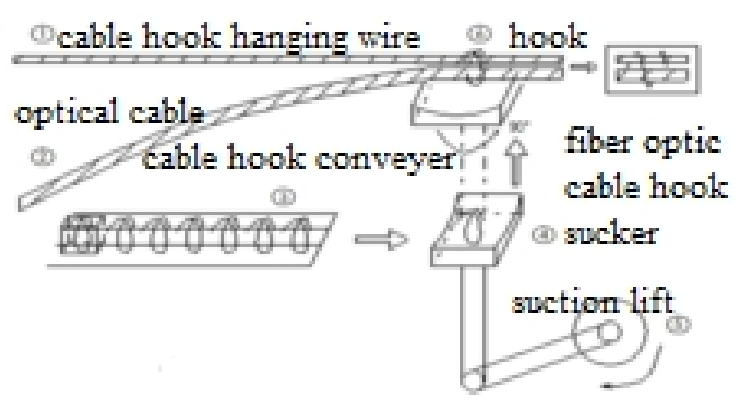

Fig.4 Schematic of optic fiber cable hook lifting mechanism 
4.Optic fiber cable hook clamping mechanism

The optic cable hook clamping plate will move downwards, and press tight the cable hook which is on the cable hanging wire, so that the cable hanging wire can go into the cable hook buckle; when the cable hook clamping wheel will press tight the cable hook again when the cable laying vehicle is moving towards.

\section{Intelligent control mechanism}

Intelligent control section will input the control program and coordinate the various mechanism actions of the cable laying vehicle according to the cable laying specification so as to make the cable laying vehicle power traction mechanism and optical fiber transmission mechanism can operate simultaneously. When the cable laying vehicle moves forward for $50 \mathrm{~cm}$, the power traction mechanism and optical fiber transmission mechanism stop the action simultaneously.

\section{Auxiliary mechanism}

The vehicle-mounted storage batteries provide power for the cable laying vehicle. The ground remote control can adjust the working status of the optic fiber cable overhead laying vehicle at any time or remote the manual laying of fiber optic cable.

\section{Precautions for Use of Optic Fiber Cable Overhead Laying Vehicle}

The suitable working environment is required to use the cable overhead cable laying vehicle, and the transport vehicle needs to load the cable tray for the simultaneous work. The road route of the laying cable hanging wire should be relatively straight and flat, so as to minimize the access barriers of the cable overhead laying vehicle. The cable tray and frame support should be loaded into the rear end of the transport vehicle compartment, which should be installed with the cable guide frame for transmission of cable to the cable laying vehicle. The transport vehicles should move slowly in the same direction as the cable laying vehicle with the cable laying process, so as to collaboratively complete automatic laying of the cables.

\section{Conclusion}

With the rapid development of optical communication technology, it is an inevitable development trend for the optic fiber cable laying mechanization and automation to gradually replace the traditional manual method. It is of great significance to improve the communication level and degree of modernization of China, and promote the rapid and orderly development of the national economy. On the basis of learning from the overhead laying winding machine technology both from home and abroad, it has become possible to develop the new cable overhead laying vehicle with the independent intellectual property rights. In this paper, the optic fiber cable overhead laying winding machine which has been put into commercial applications both home and abroad is introduced; the structure and function characteristics of the optic fiber overhead laying vehicle are analyzed and designed, and the possible problems and difficulties may be encountered when using the optic fiber cable overhead laying vehicle are discussed, hoping to provide references for the national development of optic fiber cable overhead laying vehicle. 


\section{Bibliography:}

[1].Design and Cases of Communication Engineering, Du Sishen, Electronic Industry Press, 2009 [2].The Ministry of Industry, Major Communications Capabilities of Communication Industry in the First Quarter of 2015, 2015-04-17 index number: 000019692/2015-00092

[3].Li Shuping, Optic Fiber Cable Laying Methods and Application Scenario Analysis [Journal Papers] SCIENCE AND TECHNOLOGY OF WEST CHINA, 2011,10 (6)

[4].Hu Jiayu, Liu Minghui, Analysis of Overhead Optic Fiber Cable Laying Methods [Journal Papers] HEILONGJIANG SCIENCE AND TECHNOLOGY INFORMATION, 2010 (16)

[5].Optical Fiber Cable Communication Project, Li Ligao, Posts and Telecom Press, August 2004

[6].Hui Ningli, Lu Jiancun, He Aifeng, Cao Chunqiang, Influence of Fiber Cables Bending on Transmission Efficiency of Laser Energy, [Journal Papers] ACTA PHOTONICA SINICA, 2008,37 (12)

[7].Igo Iizuka, Modes and Dispersion in OpticalFibers,DOI:10.1002/0471221376.ch11

[8].Bob Eisele, Economic \& Efficient Fiber Optic Winding: How A Producer of Winding Equipment Simplified Machine Design to Cut Costs and Conserve Space, Wire \& cable technology international: Serving Manufacturers, Specifiers and Users of Wire and Cable 2003,31(6) 